\title{
Study of the Hybrid Zone in the Mandibular Condyle in the Human Fetus
}

\author{
Wilfredo Molina Wills ${ }^{1 *}$, Elbert Reyes ${ }^{2}$ and Vanessa Rodriguez ${ }^{3}$ \\ ${ }^{1}$ PHD, Ex-Researcher of the Physiology, Department of the Medicine School of the University of Los Andes Venezuela \\ ${ }^{2}$ Professor of the Histology Department of the Medicine School of the University of Los Andes Venezuela \\ ${ }^{3}$ Private Practice, Department of the Medicine School of the University of Los Andes Venezuela \\ *Corresponding Author: Wilfredo Molina Wills, PHD, Ex-Researcher of the Physiology, Department of the Medicine School of the \\ University of Los Andes Venezuela.
}

Received: November 14, 2019; Published: November 26, 2019

DOI: $10.31080 /$ ASDS.2019.03.0711

\begin{abstract}
Aim: Compare the area occupied by the formation of blood vessels and the area of other cells involved in the osteogenesis process. Similarly, analyze the histological characteristics of the group of cells that related to ossification mechanisms.

Methods: Serially histological sections of the temporomandibular joint in sagittal plane and dyed with hematoxyline-eosine, were used to detect the presence of mesenchymal cells, osteoblasts, osteoclasts, osteoprogenitor cells and blood vessels in the hybrid area of the mandibular condyle in human fetuses.

Results: the area of angiogenesis and the area occupied by other type of cells involved in the ossification process were significantly different. Chi square test showed that the difference between these means was statistically significant (Correlation -995, x2 .991 sig $\mathrm{p}<.0001)$.

Conclusions: The area occupied by cells other than angiogenesis was considerably larger. An orderly relationship was observed between blood vessel-forming cells, mesenchymal cells and other bone-forming cells.

Keywords: Mandibular Condyle; Craniofacial Development; Condyle Ossification; Mandibular Condyle Angiogenesis
\end{abstract}

\section{Introduction}

The condyle cartilage of the mandible is associated with morphogenesis and temporomandibular joint function. Condyle cartilage is a heterogeneous tissue containing fibroblasts, osteochondral progenitor cells, and chondrocytes. The characteristics and morphological properties intermediate between bone and cartilage have allowed calling hybrid tissue [1].

During the development of the mandibular condyle, have been described two process of ossification: intramembranous and endochondral ossification. In the process of intramembranous ossification, the mesenchymal cells differentiate directly into osteoblasts; specialized cells that secrete bone matrix. The osteoblasts differentiate into osteocytes [2].
Have been also described that at ten weeks of gestation, the first mesenchymal differentiation on the cartilage of the mandibular condyle begins. The mandible is formed for membranous ossification of the mesenchymal tissue. During the interaction between epithelial and mesenchymal tissues, the mesenchymal cells form odontoblast [3].

Vascular canals, the structures of vascularized mesenchyme present in the epiphyseal and diaphyseal cartilage of bones prior to ossification [4]. It have been showed that the vascular canals may play a certain role in the process of endochondral bone formation [5]. As well as more direct interactions necessary for basic morphogenesis as bone mineralization during endochondral and intramembranous ossification is tightly coupled with blood 
vessel branching and growth (angiogenesis) [6], The variations on the angiogenesis process are basis for alterations during osteogenesis. This includes important variations in facial bone size during human evolution [7].

Blood vessel cells and bone cells communicate in many ways during development [8]. Osteoblasts are major sources of angiogenesis factor, including vascular endothelial growth factor that stimulates angiogenesis [9]. Blood vessels provide oxygen and nutrients necessary for bone formation [10].

Alterations or reduction in the number of capillaries in trabecular bone is associated with decreased bone formation [11].

The mechanisms of interaction or association between the angiogenesis process and mesenchymal tissue and the intramembranous or endochondral ossification process are unclear [12]. In order to evaluate the relationship between angiogenesis and osteogenesis at these stages of prenatal development, the hybrid growth zone of the mandibular condyle was selected for the study. The objectives of this investigation were to compare the occupied areas the formation of blood vessels and other cell groups involved in the ossification of the jaw condyle respectively. Similarly, analyze the histological characteristics of the group of cells involved in the process of ossification of the jaw condyle in the hybrid zone.

\section{Material and Methods}

\section{Surgical procedures}

The surgical procedures were previously clarified. The temporomandibular joints (TMJs) of 12 human fetuses aborted at 12 weeks of gestation were removed in one $\mathrm{cm}^{3}$. The skin tissue of the selected temporomandibular joints was removed. These blocks were separated and identified to realize the histological sections.

\section{Histological preparation}

Condyle of the mandibles of human fetuses aborted at 12 weeks of gestation was removed in blocks. These blocks were fixed in $10 \%$ stabilized neutral formalin containing $40 \%$ formaldehyde, $4.0 \mathrm{~g}$ of sodium dehydrogenate phosphate, and $6.5 \mathrm{~g}$ of disodium hydrogen phosphate at $\mathrm{Ph} 7.0$ and stored at $4^{\circ} \mathrm{C}$. The blocks were embedded in paraffin, and these paraffin-embedded blocks were sectioned serially in sagittal planes $6 \mu \mathrm{m}$ wide with a rotatory microtome. Fifth teen sections were obtained for each mandible condyle. Sections were deparaffinized and hydrated in distilled water. Hematoxylin eosin were used for detect or not the presence of mesenchymal cells, chondroprogenitor cells, osteoblasts, osteoclasts or blood vessels respectively. The protocol used was the US Armed Force Institute of Pathology phytotechnology method. Each section was cut and mounted on a previously numbered glass slide and independently assessed by members of the histological department of the Medicine School of the University of Los Andes, Venezuela; all of the observers were specialist in histology. There was no calibration with respect to their rating, but the observer's reports were similar. The selected sections were photographed and digitalized.

\section{Quantitative analysis}

Quantitative analysis was performed with serial sections for each temporomandibular joint. The total amount of cells present in the hybrid zone of each histological section was counted and measured the area occupied by these cells (area B). The surface of vessels formation (area A) was measured. It was calculated by Image J software. This method was used to analyze the percentage of angiogenesis in the whole of the study area. In all the selected cases, the average of the percentage of the groups of cells in the hybrid zone of the mandibular condyle was calculated.

\section{Morphometric analysis}

All the images were obtained through a transmitted light microscope (Kyowa Medilux-12) with video camera (motic 2000) and motic images 2.0 Multilanguage, the same Graduated object holder with metric scale standard and $400 \mathrm{x}$ magnifications were used in all histological sections. Application software for the acquisition of the differences in staining properties and morphology facilitating identification of all studied.

\section{Photomicrography study}

Each photograph was divided into two areas A: formation of blood vessels and B: other cells such as: mesenchymal, osteoblasts, osteoclasts, involved in the process of the ossification of the mandibular condyle. This area was called other cells. In both studied areas were compared and calculated the average of the percentage of the each area for each group of cells under study.

\section{Analysis of vessels formation}

Image J software (Us national institutes of health) was use in order to detect the percentage of vessel formation in relation to the whole area in all analyzed microphotographs. Only defined cell areas were selected to get a proper calibration of software, images 
of photomicrographs were taken with the same objective of the microscope and using in all the samples one bar of 30 -micron scale, the same resolution, the same optical configuration and the same size of image. To avoid the error of the measures increasing or decreasing the number of pixels, the very small distances between the forming cells from blood vessels were discarded.

\section{Statistical analysis}

Nonparametric statistical chi-square test for one-sample case was used. The distribution of cells population in the areas in study for each sample, were conceived as consisting in only two classes: A- angiogenesis area and B- area occupied by other cells. In this study the areas A and B were measured and compared. The significant difference between the two studied areas A and B of the same sample was evaluated.

No fetus used in this study had any visible evidence of developmental abnormalities or genetic disorders. To carry out this study, samples of aborted human fetuses were used in accordance with procedures approved by the department of the physiology of the Medicine School of the University of Los Andes. All the conditions of Helsinki declaration related to research in diseased human subjects were observed.

\section{Results}

The percentages obtained from the evaluation of the areas occupied by angiogenesis and the areas occupied by the presence of other cells are shown in the table. The average area for blood vessel formation was $27.17 \%$ while the average percentage of areas occupied by other cells was $72.82 \%$.

\begin{tabular}{|l|c|}
\hline A: Angiogenesis area & B: other cells \\
\hline $20,19 \%$ & $79,81 \%$ \\
\hline $18,13 \%$ & $81,87 \%$ \\
\hline $17,59 \%$ & $82,41 \%$ \\
\hline $42,60 \%$ & $57,4 \%$ \\
\hline $36,97 \%$ & $63,03 \%$ \\
\hline $42,35 \%$ & $57,65 \%$ \\
\hline $23,97 \%$ & $76,03 \%$ \\
\hline $40,71 \%$ & $59,29 \%$ \\
\hline $18,49 \%$ & $81,51 \%$ \\
\hline $20,11 \%$ & $79,89 \%$ \\
\hline $7,47 \%$ & $92,53 \%$ \\
\hline $37,57 \%$ & $62,43 \%$ \\
\hline
\end{tabular}

Table 1
Table: The table shows the relationships between the percentages of the areas occupied by the formation of the blood vessels A) and those areas where there is presence of osteoblasts, osteoclasts, mesenchymal cells or ossiform matrix, considered as an area of other cells B).

In all randomly selected photomicrographs for this study, hematopoietic cell area, it was observed as a limited area within the total histological area studied. On the surface occupied by other types of cells, no precise limits were observed. That is, osteoblast, osteoclast and mesenchymal cells respectively are not located in spaces where only a special type of cells prevails. On the contrary, these cells can be observed mixed in the same histological area (Figure 1 to 7 ).

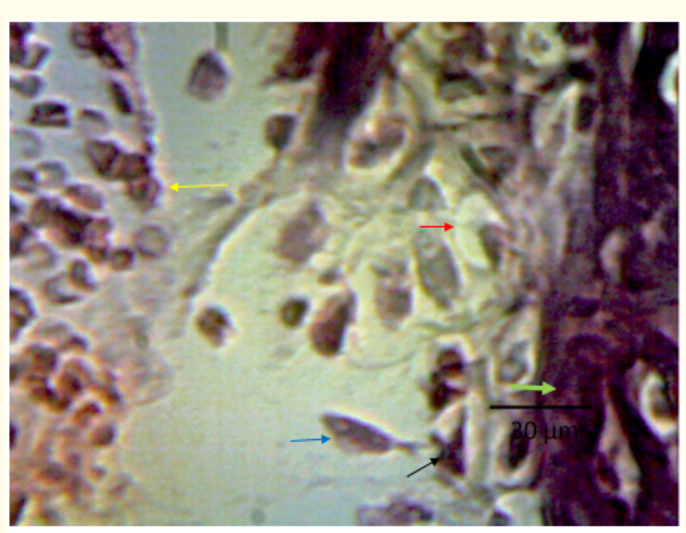

Figure 1: Area A yellow arrow, shows vessels formation. Area B black arrow, can be observe osteoblast, mesenchymal cell (blue arrow), osteoclast inside ossiform matrix (green arrow), osteoblast surrounded by matrix (red arrow).

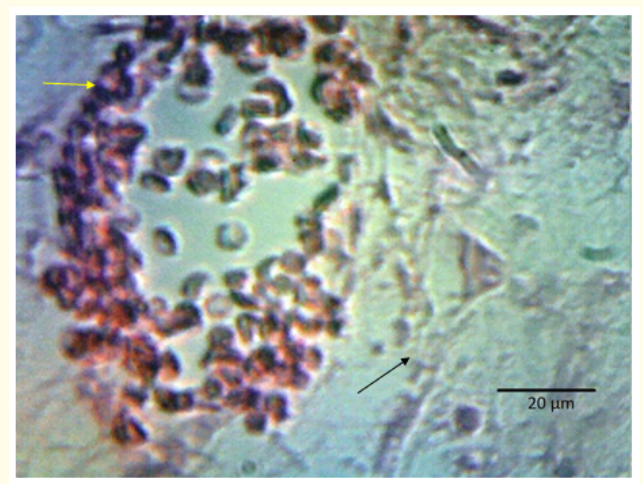

Figure 2: Area of vessels formation (yellow arrow), area of mesenchymal cells (black arrow). 


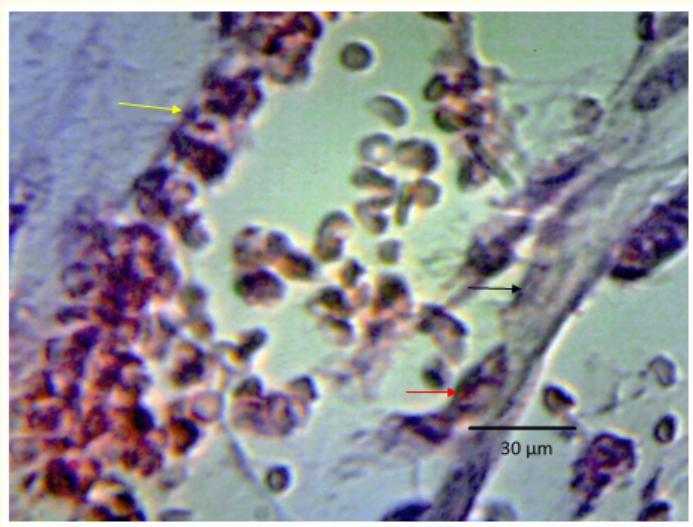

Figure 3: Area of vessels formation (yellow arrow), osteoid matrix (black arrow) and mesenchymal cell (red arrow).

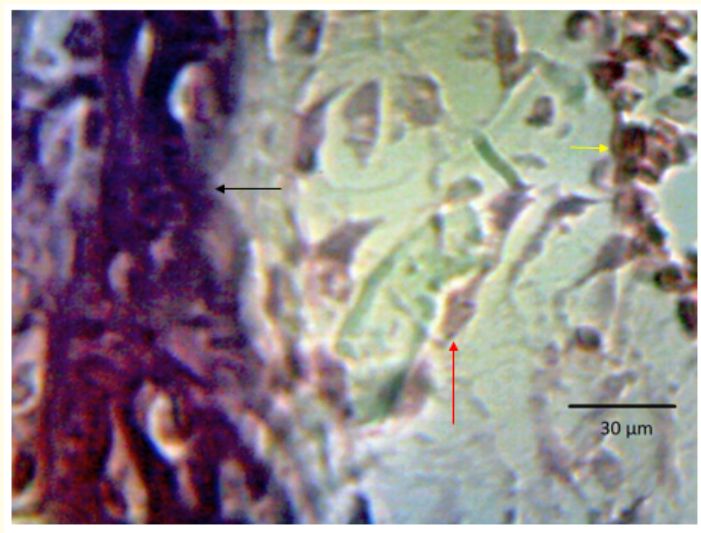

Figure 4: Vessels formation (Yellow arrow), endochondral ossification (black arrow) and mesenchymal zone (red arrow) $400 \mathrm{x}$.

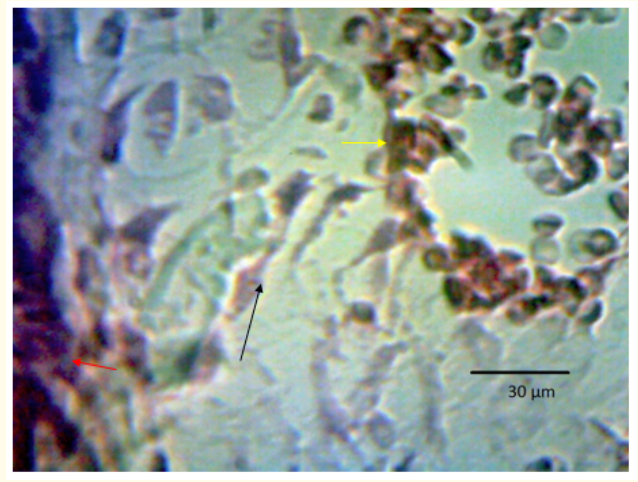

Figure 5: Angiogenesis zone (yellow arrow), mesenchymal cells zone (black arrow) and ossiform matrix zone (red arrow).

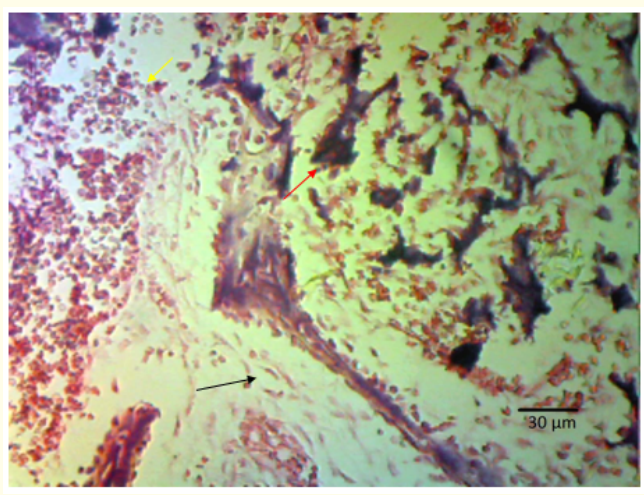

Figure 6: Angiogenesis zone (yellow arrow), mesenchymal zone (black arrow) and calcified zone (red arrow).

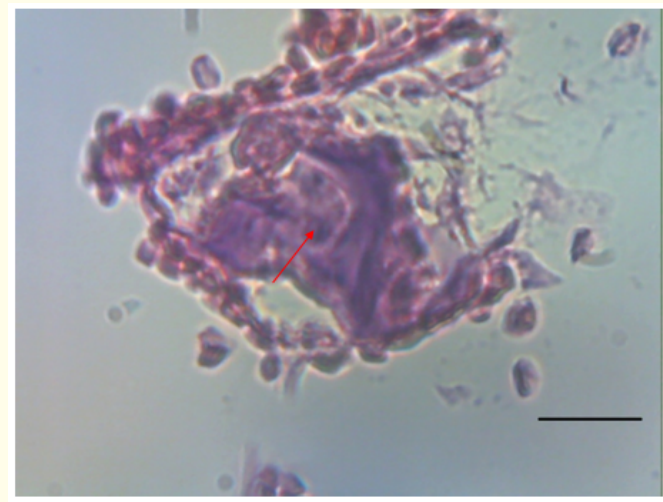

Figure 7: Osteoclast inside the matrix (red arrow) $400 \mathrm{x}$. Scale bar $30 \mu \mathrm{m}$.

In the studied histological sections, the area of angiogenesis and the area occupied by other type of cells involved in the ossification process were significantly different. Chi square test showed that the difference between these means was statistically significant (Correlation -995, $\mathrm{x}^{2} .991$ sig $\mathrm{p}<.0001$ ).

\section{Discussion}

The mandibular growth pattern was characterized by intramembranous ossification of the mandibular body and endochondral ossification of the condyle. Similary, in the early week 7 of fertilization a group of cellular mesenchymal tissue was formed around the posterior end of linear trabeculae of the mandible. In serial sections, this cellular mesenchymal tissue was traced to the fibrous mesenchyme around the Meckel cartilage [13]. 
In this study, the presence of mesenchymal cells near blood vessel-forming cells was observed in the hybrid area at these stages of gestation. Moreover, the presence of mesenchymal cells on the osteoid matrix, suggest that at these stages of prenatal development, in the hybrid area ossification is of mixed type intramembranous and endochondral. with regard to the formation of blood vessels, the presence of an erosive penetration of these cells has been described [3]. However, this was not observed in the hybrid zone. Similarly, signs of apoptosis were not observed. It has been described that angiogenesis dysregulation may contribute with craniofacial dysmorphology such as midfacial hypoplasia and craniosynostosis [14]. In this study, in all the cases studied, the zone occupied by the formation of blood vessels, was observed occupying a defined area without invasion of other tissues and closely related to the area of mesenchymal cells. For this reason, it is possible to suggest that at this stage of prenatal development the proximity between blood vessels, mesenchymal condensation and differentiation of osteoprogenitor cells are intimately related.

\section{Conclusions}

In the histological sections studied, two areas of cells were clearly observed, one of blood vessel formation and the other composed of mesenchymal cells, osteoblasts, osteoblasts surrounded by matrix and even osteoclasts within the matrix ossiform. The area occupied by cells other than angiogenesis was considerably larger. No signs of invasion or destruction of tissues were observed in the hybrid zone. An orderly relationship was observed between blood vesselforming cells, mesenchymal cells and other bone-forming cells.

\section{Acknowledgments}

The authors want to thank Dr. Augusto A Molina for his contribution.

\section{Conflicts of Interest}

The authors declare that there is no known conflict of interests associated with this publication.

\section{Bibliography}

1. Itaru M., et al. "Growth of the mandible and biological characteristics of the mandibular condylar cartilage". Japanese Dental Science Review 49.4 (2013): 139-150.

2. Rosy S and Paulus R. "Bone development and growth". (2018): 2452.

3. Merida JR., et al. "Development of the mandibular condylar cartilage in human speciments of 10-15 weeks, gestation". Journal of Anatomy 214.1 (2014): 54-64.
4. Blumer MJ., et al. "Cartilage canals in the chicken embryo are involved in the process of endochondral bone formation within the epiphyseal growth plate". The Anatomical Record 279 (2004): 692-700.

5. Blumer MJ., et al. "Identification and location of bone forming cell within cartilage canals on their couse into the secondary ossification centre (SOC)". Journal of Anatomy 208 (2006): 695-707.

6. Marks $\mathrm{O}$ and Odgren PR. "Structure and development of the skeleton". In Principles of bone biology. San Diego: academic press $202: 3-16$.

7. Dietrich EM and Antoniades K. "Bone vasculature interaction in the mandible: is bone an angiogenic tissue?" Medical Hypothesis 79.5 (2012): 582-584.

8. Clarkin CE and Gerstenfeld LC. "VEGF and bone cell signaling: an essential vessels for communication?". Cell Biochemistry and Function (2013): 1-11.

9. Hu K and Olsen BR. "Osteoblast- derived VEGF regulated osteoblast differentiation and bone repair". Journal of Clinical Investigation 126 (2016): 509-526.

10. Kusumbe AP., et al. "Coupling of angiogenesis and osteogenesis by a specific vessel subtypein bone". Nature 507 (2014): 323-328.

11. Burhard TR., et al. "Changes in trabecular bone, hematopoiesis and bone marrow vessels in aplastic anemia, primary osteoporosis and old age: a comparative histomorphometric study". Bone 8 (1987): 157-164.

12. Karaplis AC. "Embrionic development of bone and regulation of intramembranous and endochondral bone formation". In Principles of bone biology. Vol 1, 3er ed". Academic press (2008): 53-84.

13. Suk K L., et al. "Prenatal development of the human mandible". Anatomical Record. 263 (2002): 314-325.

14. Percival CJ. "The influence of angiogenesis on craniofacial development and evolution. Doctoral dissertation". University Park, PA, Penn State University (2013).

\section{Volume 3 Issue 12 December 2019 (C) All rights are reserved by Wilfredo Molina Wills., et al.}

